\title{
LA CONTRIBUCIÓN A LA PASTORAL VOCACIONAL DE LOS CONGRESOS CONTINENTALES EUROPEO Y AMERICANO
}

DOI: https://doi.org/10.52039/seminarios.v53i182.632

\begin{abstract}
Autor: Francis Bonnici. Director de la Pontificia Obra para las vocaciones Eclesiásticas de la Congregación para la Educación Católica, Vocazioni, 5 (04) 5-18.
\end{abstract}

La organización de tres Congresos Continentales sobre la pastoral vocacional en la Iglesia en estos continentes -América Latina (Itaici, Brasil, 1994), Europa (Roma, 1997) y América del Norte (Montreal, 2002)ha mostrado que mientras un programa vocacional tiene siempre puntos válidos de concreción en cada lugar del mundo, al mismo tiempo cada lugar en el que se desarrolla este programa debe estudiar el sitio concreto y la comunidad cristiana para preparar bien el ambiente donde caerá la simiente de las vocaciones al ministerio sacerdotal, a la vida consagrada y a la vida consagrada en el mundo.

La iniciativa de organizar estos Congresos Continentales ha sido asumida por la Congregación para la Educación Católica por medio de la Obra Pontificia para las Vocaciones Sacerdotales. 


\section{La solicitud de la Iglesia por la pastoral para las vocaciones al sacer- docio y a la vida consagrada}

La Iglesia católica tenía ya constituido su órgano desde hace tiempo, La Pontificia Obra para las Vocaciones Sacerdotales ${ }^{1}$ para promover las vocaciones sacerdotales en la comunidad cristiana en todo el mundo. Con el Motu Proprio Cum nobis ${ }^{2}$ Pío XII instituyó la Pontificia Obra Primaria a la que seguirán los Estatutos ${ }^{3}$ y las Normas Ejecutivas ${ }^{4}$ emanados de la Sagrada Congregación de Seminarios. La Obra se construyó sobre el esquema de las pías uniones del Código ${ }^{5}$, regido por normas del derecho común 6 ; desde el principio fueron nombrados un Presidente y un Vicepresidente, esto es, el Cardenal Prefecto y el Secretario del Dicasterio 7.

Le fue asignada la finalidad de promover las vocaciones sacerdotales en toda la Iglesia y como tarea principal tiene la facultad de agregarse personas físicas o entes de cualquier clase. La P.O.V.S. debía cuidar: las instituciones y el incremento, en las Iglesias locales, de obras específicas para promover las vocaciones; iniciar la plegaria por las vocaciones, las acciones (por ejemplo, el sostenimiento de los seminarios). Producir publicaciones. Convocar congresos.

\section{Tanto en épocas de abundancia}

El Primer Congreso Internacional fue organizado antes del Concilio Vaticano II y se fueron desarrollando más Congresos internacionales después del Concilio. ¿Para qué un Congreso Internacional para las vocaciones en 1961? Ante todo estaban las insistentes angustiosas llamadas que el Santo Padre (Juan XXIII, hoy beato) ha dirigido al mundo católico sobre el fundamental argumento de la continuidad del clero. Después el vivo interés y la diligente iniciativa a favor de las Vocaciones eclesiásti-

\footnotetext{
${ }^{1}$ RAFFAELE SACCO, Congressi Internazionali sulle vocazioni di 'Speciale' consacrazione, in Dizionario di Pastorale vocazionale, Editrice Rogate 2002, 279-280.

2 AAS 33 (1941) 479.

3 AAS 35 (1943) 369-370.

4 Ibid. 370-373.

5 Código 1918, Can 720.

6 Ibid. Can. 721.

7 Ved Normas.
} 
cas, que la Sagrada Congregación veía crecer cada vez más en todas las partes del mundo. Después estaba la proximidad de un extraordinario acontecimiento de la Iglesia Católica: el Concilio Ecuménico Vaticano II8.

En su Alocución, antes del Primer Congreso Internacional, Juan XXIII oraba: "Dona Ecclesia tua, Domine, sacerdotes sanctos; dona sacerdotes sapientiae plenos atque actuosos"9. Aún no se había manifestado la crisis numérica de las vocaciones, mas era ésta la oración que debía brotar de toda la Iglesia dirigida al Padre y que debía acompañar toda su actividad con el objetivo de aumentar el número de vocaciones para sostener la misión de la Iglesia en el periodo pre-conciliar.

Con el Concilio Vaticano II (1963-1965), la Iglesia inició su renovación de cara a prepararse para Tercer Milenio. Respecto a la renovación de la pastoral vocacional para el ministerio sacerdotal, para la vida consagrada además para la vida consagrada en el mundo, la Iglesia no quería solamente diseñar una nueva pastoral vocacional en el decreto conciliar Optatam totius (28 de octubre de 1965) ${ }^{10}$, sino que quería que la pastoral vocacional surgiese de la Eclesiología de la Iglesia-Comunión que se encontraba en el conjunto de la teología del Concilio Vaticano II'11.

${ }^{8}$ Actas del Primer Congreso Internacional. Ciudad del Vaticano 1962, 14.

${ }^{9}$ Ibid. La plegaria del Santo Padre "al dueño de la mies para que mande operarios a su viña" (Mt 9, 38; Lc 10,2) se divulgaba en toda la Iglesia con la institución de la Jornada Mundial de Oración por las Vocaciones por Pablo VI (23-01-1964). La institución de esta Jornada de oración que tiene como objeto las vocaciones sacerdotales, a la vida consagrada, y también a los institutos seculares, era el cumplimiento de lo que pedía el P. Anníbale Di Francia. El Santo de la Rogate.

${ }^{10}$ La Optatam totius señaló una nueva etapa de la pastoral vocacional: "El deber de promover las vocaciones sacerdotales compete a toda la comunidad cristiana", (n. 2); "con una vida perfectamente cristiana" (ibid.); "todos los sacerdotes demostrarán su celo apostólico sobre todo en favorecer las vocaciones, y con su vida humilde... como con el ejemplo de su mutua caridad sacerdotal..." (Ibid.); "Es obligación de los Obispos estimular la propia grey a favorecer las vocaciones y cuidar a este fin la estrecha unidad de todas las energías y de todas las iniciativas..." (Ibid.); "Esta real participación de todo el pueblo de Dios a la obra de las vocaciones corresponde a la acción de la Providencia Divina" (Ibid.). En efecto la Iglesia es llamada por Jesús: "La mies a recoger es mucha, pero los operarios son pocos. Rogad, pues, al dueño de la mies que mande obreros a su mies" (Mt 9,37-38).

11 BRUNO FORTE, La Iglesia de la Trinidad, Milán, 1998; FRANCIS BONNICI, The Solicitude of the Church for priestly formation and education, in Melita Theologica Vol.LIII/2, University of Malta 2002, 141. 


\section{Como en épocas de penuria}

En el post-Concilio, la Congregación para la Educación Católica y otros Dicasterios organizaron Congresos internacionales para los directores y otros responsables de las vocaciones de las diversas naciones. Pero también las Iglesias particulares promovían Congresos Nacionales y elaboraban planes nacionales. En el ámbito de las diócesis, como en las parroquias y en lugares como la familia, la escuela y las asociaciones y movimientos eclesiales se origina una red de actividad y plegaria por las vocaciones. Se requería una fe grande, una fuerte esperanza y un inmenso amor por la Iglesia para que todos los componentes de la Iglesia perseveraran en su acción y plegaria por las vocaciones ${ }^{12}$.

La Congregación para la Educación Católica de acuerdo con las Congregaciones para las Iglesias Orientales, para los religiosos y los Institutos Seculares y para al Evangelización de los pueblos promueve el segundo y último Congreso Internacional de los Obispos y otros responsables de las Vocaciones desde el 10 al 16 de mayo de $1981^{13}$ con el tema "Desarrollo de la atención pastoral de las vocaciones en la Iglesia particular; experiencias del pasado y programas para el futuro". El Congreso quería recoger los frutos que habían madurado en las diversas naciones del mundo y al mismo tiempo mirar al futuro.

Hacia el fin de los años ochenta, en el 1987, tienen lugar el Sínodo sobre las vocaciones y misión de los laicos en la Iglesia y en el mundo. Eran los laicos que al final de este Sínodo han pedido al Santo Padre que procure a la Iglesia sacerdotes santos para la Nueva Evangelización. Como efecto de esta petición el Sínodo sobre la Formación de los sacerdotes en las circunstancias actuales (1990).

\section{"Siempre reformanda"}

El 25 de marzo de 1992, el Santo Padre Juan Pablo II publicó su Exhortación Apostólica Post-Sinodal Pastores dabo vobis acerca de la Formación de los sacerdotes en las circunstancias actuales ${ }^{14}$.

\footnotetext{
12 Dizionario di pastorale vocazionale, Editrice Rogate 2002, 281-286.

13 Dizionario di pastorale vocazionale, Editrice Rogate, 287-289.

14 JUAN PABLO II, Exhortación Apostólica Post-Sinodal, Pastores dabo vobis (25 de marzo de 1992) AAS.
} 
En su introducción, el Santo Padre decía: "En línea con el Concilio Vaticano II acerca del Orden de los presbíteros y su formación, y deseando aplicar concretamente a las diversas situaciones esa rica y probada doctrina, la Iglesia ha afrontado en muchas ocasiones los problemas de la vida, ministerio y formación de los sacerdotes... ya en la Asamblea general, celebrada en octubre de 1967, el Sínodo dedicó cinco sesiones generales al tema de la renovación de los seminarios" 15 .

A continuación, Juan Pablo II revelaba como un secreto afirmando: "El mismo Sínodo quería responder también a una petición hecha en el Sínodo anterior que trató sobre la vocación y misión de los laicos en la Iglesia y en el mundo. Los mismo laicos habían pedido la dedicación de los sacerdotes a su formación para ser ayudados oportunamente en el cumplimiento de su común misión eclesial"16.

En la dinámica del trinomio Misterio, Comunión y Misión, encuentra Pastores dabo vobis el origen de cada llamada y el ambiente 'natural' donde nacen, se forman y maduran las vocaciones al sacerdocio ministerial para guiar a la comunidad cristiana, y las vocaciones a la vida consagrada que sirven para testimoniar la vida que vendrá, y también las vocaciones a la vida consagración en el mundo, o sea las personas llamadas por Dios a guiar a todos los componentes de la Iglesia a alcanzar la santidad dentro del Misterio de la Trinidad ${ }^{17}$.

En el marco de la formación del Presbítero, la Pastores dabo vobis afronta en el capítulo cuarto, desde el número 34 al número 41, un itinerario vocacional que concierne tanto al periodo anterior a la Ordenación como al periodo siguiente.

\section{Los Congresos Continentales y la diversidad en la concretización de la pastoral vocacional}

Tras la publicación de la Pastores dabo vobis, la Congregación para la Educación Católica, a través de la Obra Pontificia para las Vocaciones Sacerdotales, organizó tres Congresos Continentales. ¿Cuáles fueron las motivaciones para la elección continental?

\footnotetext{
15 Optattam totius, 3.

16 Ibid.

17 Pastores dabo vobis, 12.
}

.


La respuesta se encuentra en el Decreto conciliar Optatam totius, que dice: "Entre tanta diversidad de pueblos y de regiones no es posible sancionar leyes si no son de carácter general; se elabora por eso en cada nación y cada rito un particular 'Reglamento de formación sacerdotal' que deberá ser recopilado por las Conferencias Episcopales. Con tal reglamento las leyes generales vienen adaptadas a las circunstancias particulares de tiempo y lugar". En el segundo número del mismo Decreto conciliar viene diseñado un bosquejo de programa vocacional. No obstante indica que se ha de ser fiel a cuanto ha dicho de la formación sacerdotal en el primer número, o sea, que no es un programa vocacional válido para todas las áreas geográficas del mundo.

La organización de tres Congresos Continentales en la Iglesia ha mostrado que mientras un programa vocacional tiene siempre puntos válidos a concretar en cada punto del mundo, al mismo tiempo cada lugar donde se despliegue este programa debe estudiar la tierra y la comunidad cristiana local para preparar bien el ambiente donde caen las semillas de la vocación al ministerio sacerdotal, a la vida consagrada contemplativa y a la vida consagrada en el mundo.

En palabras del cardenal Pío Laghi, en aquel momento Prefecto de la Congregación para la Educación Católica, el objetivo del primer Congreso, el de América Latina era "para asentar las raíces comunes de una renovada pastoral vocacional, más específica y adecuada a la necesidad de las diferentes áreas geográficas"18.

Los Congresos Continentales han examinado la situación local no solamente de un país en particular, sino de un área geográfica más vasta, donde se encontraban grandes comunidades cristianas que tenían características sociales y eclesiales peculiares de ese área geográfica.

\section{Estudiar el terreno de la comunidad cristiana}

En el Congreso que tuvo lugar en América Latina se habló del incremento demográfico, de la amenaza de las sectas, del quinto centenario de la Evangelización del Continente y del aumento de las vocaciones ${ }^{19}$.

\footnotetext{
18 Seminarium, Anno XXXIV/3, 1994, 405.

19 PRIMER CONGRESO CONTINENTAL LATINOAMERICANO DE VOCACIONES, La pastoral vocacional en el continente de la Esperanza, 22-27 de mayo de 1994, 6 n.15. Seminarium, Año XXXIV/3, 1994, 405.
} 
En el Congreso Europeo "se completó un análisis detallado de la realidad vocacional europea... se mostró una especial predilección por los jóvenes, los valores y contravalores en los que creen, en las dificultades y aperturas que pueden obstaculizar o favorecer una propuesta vocacional"20.

En América del Norte "el desafío pastoral fundamental es el de crear una 'cultura vocacional' en la Iglesia local, o sea una cultura en la cual cada uno de los cristianos es responsable de la misión a la que es llamado como miembro de Cuerpo de Cristo"21.

\section{Proyectos a concretar en un periodo de tiempo determinado}

El Congreso de América Latina miraba a "un creciente número de ministros ordenados y de vida consagrada con la promoción de una pastoral juvenil unida a la pastoral familiar y a la catequesis para acompañar a los jóvenes en su respuesta a la llamada de Dios, con profundización en los itinerarios de formación permanente de los jóvenes en la comunidad y afianzar a aquellos que han descubierto la vocación frente a la sociedad moderna; se proyectaba también determinar instancias de colaboración e integración entre los organismos de la Iglesia"22.

También en el Congreso Europeo "se ha enfocado la pastoral juvenil como pastoral vocacional...para ayudar a que los jóvenes superen la indecisión ante las elecciones definitivas, prestando atención al problema nodal de la pastoral vocacional como el del 'discernimiento' que ha puesto en urgencia especial la actitud de cada pastor o educador de acoger la acción del Espíritu en el mundo íntimo de la persona para poder hacer una propuesta pedagógica sabia que se convierta en camino de acompañamiento de los jóvenes en busca"23.

En América del Norte el Congreso Continental afirmaba su confianza en la "realidad teológica, o sea, que cada vocación cristiana es verdaderamente un 'don de Dios' dado a favor del Pueblo de Dios... Dios continúa invitando a hombres y mujeres a vivir un compromiso permanente en la

20 Las vocaciones al sacerdocio y a la vida consagrada en Europa, 1998, 5.

21 Conversión, Discernimiento, Misión, Roma 2002, 12.

22 La Pastoral vocacional en el Continente de la Esperanza, 8; Seminarium, año XXXIV/3, 1994, 405.

23 Las vocaciones al sacerdocio y a la vida consagrada en Europa, 1998, 5-6. 
vida consagrada y en el ministerio ordenado; para realizar este diálogo entre Dios y los jóvenes (en particular) se exige de los ministros ordenados, de los consagrados y consagradas compartir con los jóvenes su propia historia espiritual y el testimonio de una Iglesia que se muestra abierta a acoger y a proporcionar espacio a sus dones y a sus energías. De esta manera los jóvenes católicos se mostrarán disponibles a acoger la invitación personal a considerar la vida consagrada y el ministerio ordenado como camino posible de recorrer para dar testimonio de Cristo en el mundo"24.

\section{Una palabra optimista dirigida a todos los destinatarios}

El Congreso de América Latina se expresó de una forma que iba más allá de su destinatarios directos, al decir que "la pastoral vocacional debe fructificar por el bien de la Iglesia universal", dirigiéndose a continuación a los niños, a los adolescentes y a los jóvenes para que tomen en consideración la llamada de Dios. A los promotores vocacionales renueva la petición de no desanimarse en "su trabajo indispensable", y a todo el pueblo de Dios le pide continuar rogando para dar testimonio con la vida"25 .

Sabedores "de la época difícil para la Iglesia en Europa, el Congreso Europeo ha buscado "un renovado impulso evangelizador de parte de la comunidad cristiana, poniendo la urgencia de la conversión y de gran esfuerzo para sostener con eficacia sobre todo la pastoral juvenil en perspectiva vocacional “. Recuerda además al Pueblo de Dios la oración de Jesús: "La mies es mucha y los operarios pocos..." (Mt 9,38). Permanece la pregunta, ¿para qué tanto trabajo en el ámbito vocacional en Europa con tan magros resultados? El Congreso Europeo no olvida "otra frase que los Apóstoles dirigen a: "Hemos faenado toda la noche, y no hemos cogido nada" (Lc 5,5)26.

El Congreso Norteamericano "extiende la invitación a los jóvenes y a los no tan jóvenes que están abiertos a explorar la posibilidad del ministerio ordenado y de la vida consagrada". Todos cuantos son invitados a concentrarse más directamente en la plegaria, la catequesis y la experien-

\footnotetext{
24 Conversión, Discernimiento, Misión, Roma 2002, 14-15.

25 Seminarium, año XXXIV/3, 1994, 643.

26 Las vocaciones al sacerdocio y a la vida consagrada en Europa, 1998, 643.
} 
cia pastoral, el acompañamiento espiritual y la llamada vocacional específica"27.

\section{El Post-Congreso para asegurar una continuidad al trabajo hecho}

En la América Latina, el Congreso pensaba "en un renovado impulso en los diversos planos de la pastoral vocacional orgánica a nivel continental, nacional, diocesano, parroquial verificada al término de un quinquenio"28.

El Congreso Europeo a su vez ponía "la petición del guía espiritual", o sea que sean "siempre figuras significativas, capaces como el Bautista de señalar al Señor, educadores que en la fe sepamos atrevernos a hacer la propuesta" porque después "El llama a quienes quiere" (Jn 15,16)29.

En América del Norte, el documento conclusivo tenía "cinco puntos principales: evangelizar o catequizar de tal modo que comunique verdaderamente el Evangelio, formar parroquias y pequeñas comunidades de fe, comprometerse en la pastoral familiar, invitar de modo explícito a los jóvenes a considerar la llamada al sacerdocio o a la vida consagrada, dar continuidad al Congreso en las parroquias, en las diócesis y en las comunidades locales" 30 .

\section{Tres Mensajes diferentes del Santo Padre en los Congresos Continen- tales}

Dirigiéndose al primer Congreso Continental, el Santo Padre llama al continente de la América Latina "el Continente de la esperanza"31, reflexionando sobre el título del Congreso: "La Pastoral vocacional en el continente de la esperanza". Este Congreso se celebraba a poca distancia de la celebración del V centenario de la Evangelización del Nuevo Continente, así la relación con la nueva evangelización venía afirmando de forma consecuente el nexo natural entre la evangelización y la salvaguardia de la dignidad del hombre en el continente latino-americano.

\footnotetext{
27 Conversión, Discernimiento, Misión, Roma 2002, 99.

28 Seminarium, año XXXIV/3 1994, 405.

${ }^{29}$ Las vocaciones al sacerdocio y a la vida consagrada en Europa, 1994, 6.

30 Conversión, Discernimiento, Misión, Roma 2002, 17.

31 La pastoral vocacional en el continente de la Esperanza, 89.
} 
"Constatamos con alegría", dice Juan Pablo II, "que en estos últimos años, en el interior de las familias cristianas profundamente arraigadas en la fe, ha surgido un mayor número de vocaciones. Los seminarios diocesanos y las comunidades religiosas han visto aumentar el número de sus miembros, cosa muy estimulante. Gracias al testimonio de una Iglesia de servicio y próxima al pueblo, el Señor ha hecho surgir hombres y mujeres deseosos de dedicar toda su vida a la causa de Cristo; y, a partir de la comunidad que deja traslucir los valores evangélicos, Ellos han multiplicado en tantos jóvenes el deseo de seguirlo más de cerca. ¡Cómo no dar gracias a Dios por esta consoladora realidad!"32.

Pero el número de vocaciones era "insuficiente para satisfacer la urgente demanda de atenciones pastorales" 33 . El Santo Padre indicó la necesidad de "desarrollar desde la infancia la dimensión vocacional de la vida bautismal" 34 , para además seguir a la juventud en su camino de maduración.

En el segundo Congreso Continental, Juan Pablo II dirigía su saludo al continente Europeo con un énfasis especial en el tema elegido por el Congreso: "Nuevas vocaciones para una nueva Europa" 35 . "El Congreso... constituye un gran signo de esperanza para la Iglesia del Continente europeo y confluye providencialmente en aquel gran río de experiencia de fe, que recuerda a Europa sus raíces cristianas y a la Iglesia la misión de anunciar a Jesucristo a la generaciones del tercer milenio"36.

Juan Pablo II es totalmente consciente de la situación precaria por falta de vocaciones al sacerdocio y a la vida consagrada en Europa y pide "un acto de esperanza en el futuro de la Iglesia en Europa; un gesto de amor hacia el pueblo de Dios del 'Viejo Continente' necesitado de personas plenamente dedicadas al anuncio del Evangelio y al servicio de los hermanos"37. La atención del Congreso Europeo se dirige principalmente a los jóvenes revelando que "son bien conocidas las dificultades que hoy hacen difícil la adhesión a la propuesta de Cristo. Entre estas: el consumismo, la visión hedonista de la vida, la cultura de la evasión, el subjetivismo exas-

\footnotetext{
32 Ibid. 91.

33 Ibid.

34 Ibid.

35 Las vocaciones al sacerdocio y a la vida consagrada en Europa, 7.

36 Ibid.

37 Ibid. 8.
} 
perado, el temor a los compromisos definitivos, una difusa carencia de proyecto ${ }^{38}$.

Hay una propuesta que contrapesa este aspecto negativo de los jóvenes de hoy y la hace el Santo Padre cuando dice "señalar en la Comunidad cristiana los recursos, las esperanzas, los valores presentes en las nuevas generaciones, ofreciendo al mismo tiempo sugerencias concretas para la elaboración, teniendo como base tales premisas, de un proyecto serio de vida inspirado en el Evangelio"39. A este respecto depende tanto del testimonio de los sacerdotes, consagrados y consagradas llamados a "ser testigos gozosos en el servicio del Reino, sabiendo bien que su vida es presencia siempre significativa cerca de los jóvenes: ésta estimula o desanima, suscita el deseo de Dios, o también constituye un obstáculo para seguirlo" 40.

En el tercer Congreso Continental, el de América del Norte (Estados Unidos y Canadá) con el tema 'Vocación: don de Dios'; el Santo Padre reconoce que la fase preparatoria del Congreso "ha implicado a las Iglesias locales y a las familias religiosas y ha vivido sus momentos más significativos durante los Congresos diocesanos y regionales" ${ }^{41}$. Resaltando además la participación de "numerosos delegados, elegidos por las diócesis y por los diversos organismos que... velan por la promoción vocacional para dedicarse a una profunda reflexión sobre la vocación sacerdotal o religiosa a la luz de los elementos bíblicos y documentos del Magisterio"42.

"Los datos muestran que algunos seminarios se están llenando de candidatos al sacerdocio, que algunas Congregaciones religiosas son ricas en vocaciones, gracias, entre otras cosas, a la fecundidad vocacional de las Comunidades y de los Movimientos eclesiales nacidos hace poco. Doy gracias al Señor por estos signos de una primavera vocacional prometedora" 43 . A pesar de este aspecto positivo, es el otro lado, o sea son "otras llamadas las que desgraciadamente distraen la mente de los jóvenes y también ideas sobre el sacerdocio y sobre el ministerio sacerdotal no conforme a la ley y a la tradición eclesial"44.

\footnotetext{
38 Ibid.

${ }^{39}$ Ibid.

40 Ibid. 9.

${ }^{41}$ Conversión, Discernimiento, Misión, 105

42 Ibid.

43 Ibid.

44 Ibid.
} 
La respuesta de Juan Pablo II es ésta: "Necesitamos crear una atmósfera apropiada a estos jóvenes. Es indispensable que nosotros seamos modelos elocuentes capaces de hacer brillar a sus ojos la grandeza y la sublimidad del sacerdocio ministerial, como la felicidad profunda que está en el donarse totalmente a Cristo para servir a la Iglesia" 45 . La responsabilidad, no obstante la comparte todo el Pueblo de Dios porque "sólo una comunidad cristiana muy comprometida en el camino de la santidad y muy decidida a afirmar el primado de lo sobrenatural y a reconocer en la liturgia 'el culmen y la fuente' de cualesquiera obra apostólica será capaz de suscitar el deseo y la alegría de ofrecerse totalmente al Señor y de cultivar las semillas vocacionales al sacerdocio y a la vida consagrada que Jesús continúa situando en el corazón de muchos jóvenes”46.

\section{Muerte y resurrección}

Quizás no por casualidad el Oslam, Boletín, junio a diciembre de 2003, del Consejo Episcopal Latinoamericano, departamento de Vocaciones y ministerios, en el Plan Global 2003-2007 y precisamente en la sección pastoral vocacional presenta el proyecto 14.1 titulado "Saldo de la pastoral vocacional, objetivo: favorecer y saldar la pastoral vocacional de modo que cada bautizado encuentre los medios necesarios para descubrir su vocación y misión en la Iglesia y en el mundo"47.

De forma detallada este proyecto será concretado en cuatro aspectos:

1. Crear un equipo latino-americano para reflexionar y sostener la pastoral vocacional.

2. Evaluar la recepción del Primer Congreso Continental para las vocaciones en América Latina y en el Caribe.

3. Sostener y desarrollar la pastoral vocacional en las diversas áreas geográficas.

4. Elaborar, publicar y distribuir materiales para la promoción de todas las vocaciones ${ }^{48}$.

\footnotetext{
45 Ibid. 106.

46 Ibid. 107.

47 CONSEJO EPISCOPAL LATINOAMERICANO, Oslam, junio a diciembre de 2003, 4.

48 Ibid.
} 


\section{Momento pasado y situación actual de las vocaciones en América} Latina

Aquí algunos datos: El personal comprometido en el apostolado de la Iglesia

\begin{tabular}{|c|c|c|c|}
\hline América del Sur & $\mathbf{1 9 9 4}$ & $\mathbf{1 9 9 7}$ & $\mathbf{2 0 0 2}$ \\
\hline Clero diocesano & 20.856 & 22.341 & 25.458 \\
\hline Clero Religioso & 18.159 & 17.779 & 17.502 \\
\hline Religiosas & 91.457 & 89.888 & 88.083 \\
\hline $\begin{array}{c}\mathrm{N}^{\circ} \text { de ordenaciones del clero } \\
\text { diocesano }\end{array}$ & 894 & 891 & 973 \\
\hline $\begin{array}{c}\text { Número de candidatos al sacer- } \\
\text { docio diocesano (Filos./teol.) }\end{array}$ & 11.330 & 12.028 & 14.353 \\
\hline $\begin{array}{c}\mathrm{N}^{\circ} \text { candidatos sacerdocio } \\
\text { (religiosos) }\end{array}$ & 6.478 & 7.381 & 8.025 \\
\hline
\end{tabular}

El junio pasado recibí "Jeunes et Vocations", la revista de pastoral vocacional del Servicio Nacional de Vocaciones, París, mayo 2004. La revista da un balance de la situación vocacional en cada una de las naciones y también un artículo de Rainer Birkenmaier "Les debuts d'une collaboration européenne", el Rvdo. Birkenmaier afirma que "el documento final del Congreso Europeo de vocaciones (1977) se ha convertido en una carta de la pastoral vocacional del continente europeo"49.

El Revdo. Birkenmaier dice también que participando en el Congreso Norteamericano del 2002, se ha dado cuenta más claramente de que, si bien hay algunas semejanzas entre Europa y los Estados Unidos de América, existen también grandes diferencias:

1. Tenemos muchas naciones con lenguas y culturas diversas,

2. Nuestras sociedades se han desarrollado de maneras muy diferentes

49 RAINER BIRKENMAIER, Les debuts d'une collaboration européenne, en Jeunes et Vocations, 113, mayo de 2004, 9. 
3. La posición de la Iglesia es muy diversa en las diferentes naciones,

4. El clima vocacional es distinto de una nación a otra ${ }^{50}$.

Tras una reflexión sobre la necesidad de evangelización del continente europeo, Rainer Birkenmaier concluye que "en Europa, la pastoral vocacional se encuentra en situación problemática" ${ }^{51}$. Y esto por dos causas:

1. "En la Iglesia local de Europa, los caminos tradicionales de cristianización han perdido su eficacia, y

2. la Iglesia de Europa no es ya una Iglesia que evangeliza y llama, sino que en la mayor parte de las naciones europeas la pastoral vocacional sufre de cierta carencia de claridad y de un crónico estancamiento" 52.

\section{Momento pasado y situación actual de las vocaciones en Europa}

Presentamos algunos datos: el personal comprometido en el apostolado de la Iglesia

\begin{tabular}{|c|c|c|c|}
\hline Europa & $\mathbf{1 9 9 4}$ & $\mathbf{1 9 9 7}$ & $\mathbf{2 0 0 2}$ \\
\hline Clero diocesano & 152.169 & 148.595 & 141.724 \\
\hline Clero Religioso & 66.412 & 64.803 & 62.027 \\
\hline Religiosas & 414.687 & 388.693 & 348.085 \\
\hline $\begin{array}{c}\mathrm{N}^{\circ} \text { de ordenaciones del clero } \\
\text { diocesano }\end{array}$ & 2.493 & 2.458 & 2.118 \\
\hline $\begin{array}{c}\text { Número de candidatos al sacer- } \\
\text { docio diocesano (Filos./teol.) }\end{array}$ & 19.802 & 18.618 & 16.207 \\
\hline $\begin{array}{c}\mathrm{N}^{\circ} \text { candidatos sacerdocio } \\
\text { (religiosos) }\end{array}$ & 9.709 & 9.235 & 8.816 \\
\hline
\end{tabular}

\footnotetext{
50 Ibid. 10.

51 Ibid. 11.

52 Ibid.
} 
Su Excelencia Mons. Kevin M. Britt ${ }^{53}$ ha escrito a la Obra Pontificia para las Vocaciones Sacerdotales indicando que en los Estados Unidos está en fase de actualización el Plan Pastoral del Tercer Congreso Continental para las vocaciones al Sacerdocio ministerial y a la Vida consagrada. El 12 de mayo de 2004 era el último día posible para enviar sugerencias o planes de acción desde los Congresos diocesanos organizados en 2003. Con estas sugerencias la Comisión Episcopal para las vocaciones elaborará un plan nacional para las vocaciones en los Estados Unidos que se llamará "Future Hope of the Church" a la que está llamada a colaborar también la Obra Pontificia para las Vocaciones Sacerdotales, que está colaborando en la redacción del plan arriba mencionado.

Momento pasado y situación actual de las vocaciones en América del Norte

Presentamos algunos datos: el personal comprometido en el apostolado de la Iglesia

\begin{tabular}{|c|r|r|r|}
\hline América del Norte & $\mathbf{1 9 9 4}$ & $\mathbf{1 9 9 7}$ & $\mathbf{2 0 0 2}$ \\
\hline Clero diocesano & 39.195 & 38.215 & 36.676 \\
\hline Clero Religioso & 23.220 & 22.088 & 20.308 \\
\hline Religiosas & 122.670 & 113.010 & 96.041 \\
\hline $\begin{array}{c}\mathrm{N}^{\circ} \text { de ordenaciones del clero } \\
\text { diocesano }\end{array}$ & 468 & 474 & 435 \\
\hline $\begin{array}{c}\text { Número de candidatos al sacer- } \\
\text { docio diocesano (Filos./teol.) }\end{array}$ & 3.937 & 3.678 & 3.910 \\
\hline $\begin{array}{c}\mathrm{N}^{\circ} \text { candidatos sacerdocio } \\
\text { (religiosos) }\end{array}$ & 1.755 & 1.647 & 1.721 \\
\hline
\end{tabular}

${ }^{53}$ Desgraciadamente poco después falleció S. E. Mons. Britt. 
¿Cuál sería necesario para el eventual resurgir de la pastoral vocacional en los tres continentes? "Se trata de la esencial transmisión de la verdad de la fe, indispensable para el nacimiento y crecimiento de una comunidad de discípulos, que a su vez se convertirán en testimonios". Esta respuesta viene del Cardenal Jean Pierre Schotte cuando comenta sobre "Los Sínodos Continentales para una nueva Evangelización al comienzo del tercer milenio" 54 .

A nivel continental se han celebrado Sínodos de Obispos: de Europa, África, América, Asia, Oceanía. Estos Sínodos invitan a confesar a Jesucristo que ofrece una esperanza contra toda esperanza y a operar sobre su enseñanza una atenta y sapiencial lectura del tiempo presente portador de signos y semillas de esperanza" 55 . Una vez más, la gracia de la evangelización es la misma para todos en todos los continentes. El Cardenal Schotte insiste en que esta gracia "aparece en cada uno de estos Sínodos de modo adecuado a las condiciones propias de cada continente, ya que estas asambleas constituyen ya por sí mismas parte de la nueva evangelización" 56 .

Basta con seguir las directivas que el Santo Padre da respecto a la pastoral vocacional en las diversas exhortaciones Apostólicas post-Sinodales ${ }^{57}$, para tener la justa inspiración para una pastoral vocacional eficaz en los respectivos continentes.

54 Seminarium, XLI/2, 2001, 337.

55 Ibid.

56 Ibid. 340.

57 Ecclesia in America,22.1.1999, n. 40, Ecclesia in Europa, 28.6.2003, nn. 39-40. 
EL PERSONAL COMPROMISO EN EL APOSTOLADO DE LA IGLESIA

\begin{tabular}{|c|c|c|c|}
\hline & 1994 & 1997 & 2002 \\
\hline $\begin{array}{l}\text { Clero diocesano } \\
\text { Sudamérica } \\
\text { Europa } \\
\text { Norteamérica }\end{array}$ & $\begin{array}{r}20.856 \\
152,169 \\
39.195\end{array}$ & $\begin{array}{r}22.341 \\
148.595 \\
38.215\end{array}$ & $\begin{array}{r}25.458 \\
141.724 \\
36.676\end{array}$ \\
\hline $\begin{array}{l}\text { Clero Religioso } \\
\text { Sudamérica } \\
\text { Europa } \\
\text { Norteamérica }\end{array}$ & $\begin{array}{l}18.159 \\
66.412 \\
23.220\end{array}$ & $\begin{array}{l}17.779 \\
64.803 \\
22.088\end{array}$ & $\begin{array}{l}17.502 \\
62.027 \\
20.308\end{array}$ \\
\hline $\begin{array}{l}\text { Religiosas } \\
\text { Sudamérica } \\
\text { Europa } \\
\text { Norteamérica }\end{array}$ & $\begin{array}{r}91.457 \\
414.687 \\
122.670\end{array}$ & $\begin{array}{r}89.888 \\
388.693 \\
113.010\end{array}$ & $\begin{array}{r}88.083 \\
348.085 \\
96.041\end{array}$ \\
\hline $\begin{array}{l}\mathrm{N}^{\circ} \text { de ordenaciones del clero } \\
\text { diocesano } \\
\text { Sudamérica } \\
\text { Europa } \\
\text { Norteamérica }\end{array}$ & $\begin{array}{r}894 \\
2.479 \\
468\end{array}$ & $\begin{array}{r}891 \\
2.458 \\
474\end{array}$ & $\begin{array}{r}973 \\
2.118 \\
435\end{array}$ \\
\hline $\begin{array}{l}\text { Número de candidatos al sacer- } \\
\text { docio diocesano (Filos./teol.) } \\
\text { Sudamérica } \\
\text { Europa } \\
\text { Norteamérica }\end{array}$ & $\begin{array}{r}11.330 \\
19.802 \\
3.937\end{array}$ & $\begin{array}{r}12.028 \\
18.618 \\
3.678\end{array}$ & $\begin{array}{r}14.353 \\
16.207 \\
3.910\end{array}$ \\
\hline $\begin{array}{l}\mathrm{N}^{\circ} \text { candidatos sacerdocio } \\
\text { (religiosos) } \\
\text { Sudamérica } \\
\text { Europa } \\
\text { Norteamérica }\end{array}$ & $\begin{array}{l}6.478 \\
9.709 \\
1.755\end{array}$ & $\begin{array}{l}7.381 \\
9.235 \\
1.647\end{array}$ & $\begin{array}{l}8.025 \\
8.816 \\
1.721\end{array}$ \\
\hline
\end{tabular}

